\title{
PAS Rising Threat to Safe Motherhood
}

\section{Preeti Sharma*}

Cocoon Hospital, India

*Corresponding Author: Preeti Sharma, Cocoon Hospital, India.
Received: January 11, 2021

Published: January 28, 2021

(C) All rights are reserved by Preeti Sharma.
Placenta accreta spectrum is a potentially life-threatening pregnancy complication that occurs in approximately 3 in 1000 pregnancies. The incidence of this iatrogenic disorder is ever increasing with the alarming rates of caesarean deliveries and fertility enhancing surgeries in the last decade. It occurs when the placenta grows too deeply into the wall of the uterus and is unable to detach at childbirth. The etiology is a defect of the endometrial -myometrial interface (absence of fibrinoid Nitabuchs layer) that leads to a failure of normal decidualization in the area of a uterine scar, which allows abnormally deep placental anchoring villi and trophoblast invasion. FIGO has given a classification of PAS in 2019 according to the clinical symptoms and degree of adherence (Luke's classification) into creta accreta and percreta. WHO has used the wider term Morbidly adherent placenta and used code ICD 10042 for international coding.

Women with this condition face complex pregnancies and deliveries. Risks to mother and baby include

- Second or third trimester bleeding

- Excessive blood loss at delivery

- Premature delivery

- Need for a hysterectomy

- Maternal mortality.

It is a matter of considerable concern that most women with placenta accreta spectrum typically have no symptoms. In some cases bleeding may occur in the second or third trimester. Some women report pain that is much greater than expected in normal pregnancy. Often it is detected during routine sonogram or more catastrophic presentation is during routine caesarean when it is detected unexpectedly. Early antenatal diagnosis is critical because it provides an opportunity to optimize management and outcomes.

Diagnosis is usually made through ultrasound imaging. The ultrasound testing can evaluate the location of the placenta and look for signs of abnormal growth into the uterine wall specially in a previous caesarean with anterior placenta.

Although rare, cesarean scar pregnancy may be diagnosed in the first trimester and is strongly associated with subsequent placenta accreta spectrum if untreated. This occurs when the gestational sac is embedded in the uterine window (nick) at the site of a cesarean scar. The risk of placenta accreta spectrum approaches $100 \%$ if the pregnancy is allowed to continue. Other first trimester features of placenta accreta spectrum visible on sonography include a gestational sac that is located in the lower uterine segment and the presence of multiple irregular vascular spaces within the placental bed.

Magnetic resonance imaging (MRI) is the other major tool used for the antenatal diagnosis of placenta accreta spectrum. It is a complimentary tool to ultrasound and is particularly useful in diagnosing invasion in posterior and lateral placenta and obese patients and assessing depth of invasion in obese patients.

Placenta accreta can thus be expected (diagnosed earlier) or unexpected (detected intraoperative) Vertically running vessels over a bulging lower uterine segment is a classical clinical sign of placenta accreta. Incision to deliver the baby should therefore be always above this. It's management can be nonconservative with hysterectomy either immediate or delayed as per the condition of the patient and facilities available. It is prudent to close the uterus after delivering the baby with no attempt to remove the placenta and either proceed with hysterectomy or do pelvic packing and call for help or transfer the patient to a higher Center. 
Caesarean hysterectomy is the gold standard of treatment along with internal artery ligation. It requires a multispeciality team in a tertiary care centre with high end ICU and NICU and blood bank prepared for a massive haemorrhage protocol after a detailed informed consent. If detected late during antenatal sonogram or even during a caesarean it is safer to transfer the patient to a well equipped facility to deal with adherent placenta.

If fertility is to be preserved one can consider saving the uterus but risk: benefit ratio must be well considered in each case on individual basis as this treatment is still under research.

Conservative and expectant management to save the uterus include placental extirpation and Triple $\mathrm{P}$ procedure and leaving placenta behind to be removed later by Hysteroscope or delayed hysterectomy. It must be emphasised that all of these conservative and expectant methods are fraught with major risks of sepsis DIC haemorrhage and even recurrence in future pregnancies.

Methotrexate use has no scientific basis as it acts only on proliferating trophoblast tissue but is still used routinely in many cases of retained products of conception.

Tranexemic acid has been found to be useful in decreasing blood loss in cases of placenta accreta although it's routine use is not recommended in routine caesarean deliveries.

As forewarned is forearmed it is essential to look carefully in all previous caesarean pregnancies with anterior placenta. Also all practising obstetricians should be aware of this possibility and be prepared with an emergency protocol to deal with PAS to save maternal lives.

A detailed sonogram is a must in all previous caesarean cases specially if the placenta is anterior. If done early in first trimester patient can have a choice of abortion in case of caesarean scar pregnancy. Elective Caesarean at 34 weeks in a tertiary care centre should be opted in all symptomatic patients ( bleeds) of placenta accreta. Rarely a pregnancy may be allowed to continue to 36 or 37 weeks under close observation [1-8].

\section{Conclusion}

- Primary prevention is decreasing the incidence of caesarean section careful suturing technique of repair of uterus in caesarean as well as myomectomy and other reconstructive surgeries involving the uterus.
- Avoiding excess curettage during D\&E. Respect all tissues during surgeries of uterus.

- Early detection supervised antenatal care and planned delivery in a tertiary care centre are the keys to save the mother the uterus.

\section{Bibliography}

1. ACOG Committee on Obstetric, Practice. "ACOG Committee opinion. Number 266, January 2002: placenta accreta". Obstetrics and Gynecology 99.1 (2002): 169-170.

2. Hobbins John C. Obstetric ultrasound: artistry in practice. Oxford: Blackwell (2007): 10.

3. Steven G Gabbe., et al. "Obstetrics: normal and problem pregnancies (4. ed.)". New York, NY [u.a.]: Churchill Livingstone (2002): 519.

4. Bowman ZS., et al. “'Interobserver variability of sonography for prediction of placenta accreta" (2014).

5. Turrentine John E. "Clinical protocols in obstetrics and gynecology (3rd ed.)”. London: Informa Healthcare (2008): 286.

6. Committee On Obstetric, Practice. "Placenta accreta Number 266, January 2002 Committee on Obstetric Practice". International Journal of Gynecology and Obstetrics 77.1 (2002): 77-78.

7. Committee on Obstetric Practice. "Placenta Accreta". American College of Obstetricians and Gynecologists. Archived from the original on 2016-11-23.

8. Silver RM., et al. "Maternal morbidity associated with multiple repeat cesarean deliveries". Obstetrics and Gynecology 107.6 (2006): 1226-1232.

\section{Assets from publication with us}

- Prompt Acknowledgement after receiving the article

- Thorough Double blinded peer review

- Rapid Publication

- Issue of Publication Certificate

- High visibility of your Published work

Website: www.actascientific.com/

Submit Article: www.actascientific.com/submission.php Email us: editor@actascientific.com

Contact us: +919182824667 